Annuaire suisse de politique de développement

27-2 | 2008

Migration et développement: un mariage arrangé

\title{
Migration et développement : une réflexion menée par la Fédération genevoise de coopération
}

Yanik Marguerat et Christelle Genoud

\section{(2) OpenEdition}

1 Journals

Édition électronique

URL : http://journals.openedition.org/aspd/517

DOI : 10.4000/aspd.517

ISSN : 1663-9669

Éditeur

Institut de hautes études internationales et du développement

\section{Édition imprimée}

Date de publication : 1 décembre 2008

Pagination : 239-244

ISBN : 978-2-940415-07-6

ISSN : $1660-5934$

\section{Référence électronique}

Yanik Marguerat et Christelle Genoud, « Migration et développement : une réflexion menée par la

Fédération genevoise de coopération », Annuaire suisse de politique de développement [En ligne], 27-2 I 2008, mis en ligne le 16 mars 2010, consulté le 08 septembre 2020. URL : http:// journals.openedition.org/aspd/517; DOI : https://doi.org/10.4000/aspd.517 


\title{
Migration et développement: une réflexion menée par la Fédération genevoise de coopération
}

\author{
Yanik Marguerat et Christelle Genoud*
}

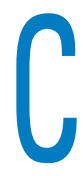

es dernières années, de nombreuses instances internationales ont travaillé et réfléchi sur l'interaction complexe existant entre la migration et le développement. On peut citer notamment le dialogue de haut niveau mis en place par les Nations unies en 2005 ou encore les rencontres du Forum mondial sur la migration et le développement (Bruxelles en 2007 et Manille en 2008). Certaines grandes organisations non gouvernementales (ONG) suisses ont aussi entamé un processus de réflexion autour de la question (Croix-Rouge suisse et Caritas notamment ${ }^{1}$ ) mais dans l'ensemble, la société civile en Suisse a encore peu exploré cette zone de recherche. Toute cette réflexion doit aussi être perçue à la lumière de la situation politique en Suisse, où les associations de migrants, leurs membres et la société civile en général opèrent actuellement dans un cadre législatif plus dur et restrictif à l'encontre des réfugiés et des étrangers extra-européens.

C'est dans ce contexte que la Fédération genevoise de coopération (FGC), un organisme faîtier regroupant 48 ONG œuvrant dans le domaine de la coopération au développement et de la sensibilisation, a choisi de travailler avec ses membres sur cette thématique ${ }^{2}$. D'une part elle a mis sur pied un événement sur trois jours, le $2^{\mathrm{e}}$ Carrefour genevois de la solidarité, qui a porté sur ce thème. La volonté de la FGC a été de mettre en avant les voix du Sud, ce qu'elle a fait en invitant à s'exprimer plusieurs ressortissants des pays du Sud qui vivent la réalité de la migration quotidiennement, notamment divers partenaires de l'Afrique de l'Ouest. En parallèle, une étude qualitative a été réalisée sur les liens existant entre la migration et les projets de coopération au développement de ses associations membres. Cette démarche s'insère dans un processus de capitalisation des expériences, dont une des étapes fut l'organisation d'un forum interne de

* Yanik Marguerat, chargé de communication, Fédération genevoise de coopération.

Christelle Genoud, économiste, sur mandat de la Fédération genevoise de coopération.

1 Bettina Zeugin, Où en est la politique migratoire de la Suisse?, prise de position, Secteur études, Lucerne, Caritas Suisse, mai 2007, <http://www.caritas.ch>, et Jean-François Giovannini (membre du Conseil de la Croix-Rouge), discours d'introduction à la conférence annuelle de la Croix-Rouge suisse intitulée «La migration, une contribution au développement?», 30 novembre 2006, $<$ http://www.redcross.ch>. Ladite conférence a débouché sur une publication: Schweizerisches Rotes Kreuz (Hrsg.), Migration, ein Beitrag zur Entwicklung ?, Zürich, Seismo, 2007.

2 La FGC et ses associations membres travaillent avec des ONG partenaires au Sud pour combattre les inégalités et augmenter la liberté de choix des sociétés et des peuples dans leur construction d'un mieux-être économique, social et culturel. Elles sont très diverses au niveau de leurs objectifs, de leurs structures et de leurs activités mais partagent toutes l'optique d'un développement global durable, basé sur les valeurs de justice sociale, d'équité et de dignité humaine. Pour en savoir plus: $<$ http://www.fgc.ch>. 
discussion entre les membres de la fédération, qui ont discuté des résultats de l'étude. Une étape ultérieure sera la parution du compte rendu du Carrefour de la solidarité, disponible sur le site Internet de la FGC ${ }^{3}$. Tous ces éléments se conjuguent, posent le contexte et viennent compléter la partie centrale de cette démarche qui est l'étude réalisée sur les projets soutenus par la Fédération et dont une partie des résultats est exposée ci-après ${ }^{4}$.

\section{Quelques résultats de l'étude «Migration et projets de coopération »}

Un premier résultat important, et indirect, de l'étude est d'avoir permis une sensibilisation à la thématique de la migration auprès des responsables de projet interrogés ici, à Genève, et de leurs partenaires au Sud. En complétant les questionnaires distribués dans le cadre de l'étude, les acteurs précités se sont en effet mis au fait de l'intérêt actuel porté par exemple aux transferts de fonds des migrants vers les pays en développement ou encore aux actions des diasporas. D'autres ont pris connaissance, via l'étude, du concept de la migration tel que défini par l'Organisation internationale pour les migrations (OIM), et qui englobe non seulement les déplacements volontaires des travailleurs mais également les déplacements «forcés», comprenant donc les personnes réfugiées, déplacées ou victimes de trafics. Le choix d'inclure aussi dans cette réflexion le phénomène de l'exode rural et les migrations temporaires liées à la saison sèche a permis de faire des liens avec de nombreux projets soutenus, liens qui n'étaient peut-être pas identifiés dès le départ par les responsables.

Un autre résultat intéressant qui est ressorti de l'étude est relatif à l'appropriation du thème par les associations et leurs membres. En effet, on peut se demander pourquoi les personnes qui pilotent les projets de développement ne se sont pas mieux approprié la thématique de la migration; une des explications réside peutêtre dans la surmédiatisation des phénomènes liés à la migration Sud-Nord. Les images dramatiques de Ceuta et Melilla ou encore des naufragés des pirogues tentant de rejoindre les côtes de l'Europe cachent une autre réalité beaucoup moins visible mais tout aussi dérangeante, à savoir que $80 \%$ environ des réfugiés ${ }^{5}$ et plus de $60 \%$ des migrants vivent dans les pays en voie de développement ${ }^{6}$.

La migration internationale est un phénomène complexe et multifactoriel qui a de nombreuses incidences sur notre monde. Lorsqu'elle est considérée dans sa définition exhaustive et qu'elle est bien explicitée, les responsables de projet qui œuvrent avec les pays du Sud sont mieux à même de la conceptualiser, la comprendre et la prendre en considération dans leurs actions, ce que l'étude menée par la FGC a permis de mettre en lumière.

$3<$ http://www.fgc.ch/carrefours.php $>$.

4 L'étude est basée, d'une part, sur des entretiens qualitatifs avec des responsables de projet en Suisse ainsi qu'avec des partenaires sur le terrain et, d'autre part, sur les données récoltées par le biais de 30 questionnaires renvoyés par les associations membres de la FGC.

5 Le nombre de réfugiés dans le monde est tombé de 18,5 millions en 1990 à 13,5 millions en 2005. Les pays en développement abritent 10,8 millions de réfugiés, dont 7,8 millions en Asie et 3 millions en Afrique (Assemblée générale des Nations unies, Migrations internationales et développement, rapport du secrétaire général, A/60/871, mai 2006, p. 46).

6 Catherine Wihtol de Wenden, Atlas des migrations dans le monde, Paris, Autrement, 2005. 
En définitive, cette appropriation pourrait être le moyen de mettre en œuvre des projets plus efficaces, viables et adaptés à une situation socio-économique donnée puisqu'ils prendraient en compte le facteur complexe de la migration.

Bien que l'étude ait relevé un certain manque d'appropriation de la thématique de la migration, certains des projets analysés, qui ont bien intégré la dimension de la migration dans le développement, contiennent des enseignements très pertinents.

Le premier exemple concerne un projet mené par l'association «Les Jardins de Cocagne - Solidarité Nord et Sud», destiné à appuyer les initiatives de développement et le renforcement de la société civile au sein de la commune de Kothiary, au Sénégal ${ }^{7}$. Cette commune compte un nombre important d'émigrés, établis notamment en France, et elle a intégré dans son Plan local de développement (PLD) la collaboration avec des migrants. Par conséquent, des représentants des autorités locales et de la société civile ainsi que des collaborateurs des Jardins de Cocagne et des membres de l'association des ressortissants de Kothiary en France se réunissent ici, au Nord, ou au village pour agir de concert. Ils ont d'ailleurs pris conscience de l'importance de mettre en place des outils de communication, avec des échanges réguliers entre le Nord et le Sud. Un exemple concret de cette collaboration est le transfert de fonds par les migrants établis en France dans les caisses locales d'épargne et de crédit, créées grâce au soutien de l'association. Le projet permet également de créer des boutiques communautaires pour lutter contre les prix excessifs des commerçants, de constituer des banques de semences et d'investir dans les domaines de l'eau, de l'éducation et de la santé. Ces nouvelles pratiques rompent avec le passé, quand les fonds envoyés par les migrants servaient aussi bien à la construction de postes de santé et de routes qu'à des dépenses improductives (maisons, élevage de prestige).

Le second exemple traite de l'expérience réalisée avec des ressortissants de la région des Grands Lacs, qui a débouché sur l' «Initiative de Genève pour la paix dans les Grands Lacs ${ }^{8}$. Cette initiative prend la forme d'un réseau de leaders issus du Burundi, du Nord- et du Sud-Kivu et du Rwanda. Un séminaire, organisé en 2003, dont l'objectif était de parvenir à dégager des pistes d'action concrètes de promotion de la paix est à l'origine de la création de ce réseau. L'intérêt premier de l'organisateur du séminaire était que les membres de la diaspora eux-mêmes apaisent les tensions au lieu de les exacerber. Selon lui, il était indispensable d'engager des actions auprès des émigrés de cette région et avec eux. C'est surtout le long processus d'identification des participants au séminaire qui est exemplaire: le responsable du projet, lui-même Rwandais établi à Genève, a rencontré de nombreuses personnes pour identifier les participants et ce afin de s'assurer de leur réelle capacité à dépasser les tensions. Leur rencontre est ainsi l'aboutissement de deux années de concertation. C'est ensuite la qualité des échanges entre les personnes réunies qui a été essentielle dans cette expérience. Les membres ont en effet témoigné de leurs différents vécus avant d'élaborer des stratégies d'action communes. Cet échange de témoignages a

7 Voir également, dans ce volume, l'entretien avec Ibrahima Thioye, un partenaire mauritanien des Jardins de Cocagne.

8 Ce projet a été mené par la Commission Tiers-Monde de l'Eglise catholique et Eirene Suisse. 
permis de créer un lien social fort entre eux, lien qui perdure cinq ans après leur première rencontre et qui a mené à la mise en œuvre de projets de développement par le réseau même. On peut citer par exemple l'organisation récente d'un forum réunissant cette fois la jeune diaspora des Grands Lacs au Sénégal, ou encore la diffusion des témoignages livrés en 2003, sous forme d'exposition, dans les villes principales des Grands Lacs9.

\section{Travailler avec les migrants?}

Les expériences de ces deux ONG avec des migrants sont intéressantes pour d'autres et il apparaît important aujourd'hui de tirer les leçons apprises de certaines initiatives. Des données actuellement collectées en Suisse faciliteront aussi ce type de collaboration. On peut citer les informations récoltées relatives à plusieurs communautés présentes en Suisse et à leur participation au développement de leurs pays d'origine ${ }^{10}$ ou encore la constitution de bases de données répertoriant les migrants qualifiés issus des pays en développement et présents en Suisse ${ }^{11}$. Tous ces outils permettront, à terme, de mieux équiper les associations au Nord souhaitant travailler avec des migrants disposant de connaissances et d'expertises pertinentes.

De nombreuses personnes migrantes vivent à Genève: travailleurs, réfugiés politiques ou économiques, fonctionnaires internationaux, personnes sans statut légal. Elles constituent un réservoir de compétences et d'expertise qui peut offrir aux organisations de développement un potentiel intéressant, à la fois pour la vie associative et pour l'élaboration de projets.

Certaines ONG membres de la FGC estiment que l'intégration des migrants en leur sein est importante. Dans le cas précis de l'Initiative de Genève pour la paix dans les Grands Lacs, tout le processus de définition et la mise en route du projet ont été pensés par des ressortissants de la région. En cela, il s'agit d'un exemple réussi de partenariat égalitaire, mais il faut aussi accepter que cette démarche de partenariat comporte des risques; si, dans ce cas précis, la migration semble représenter un potentiel, elle suppose également par la suite un partage du pouvoir, non seulement sur le plan financier, mais encore dans la manière de penser les projets.

Il est toutefois intéressant de noter que, dans quelques contextes spécifiques, certaines associations de développement travaillant à Genève ont choisi de ne pas confier le suivi de projets à des responsables issus des pays mêmes où les projets se déroulent. En effet, l'expérience leur a montré que le décalage entre

9 La démarche a en effet abouti à la publication d'un ouvrage et à la création d'une exposition, Dépasser la haine, construire la paix: un choix, un engagement, autour de cette thématique à partir des expériences vécues et relatées par des ressortissants de la région des Grands Lacs engagés dans la promotion de la paix et du «mieux vivre ensemble».

10 A savoir, une étude réalisée par le Secrétariat d'Etat à l'économie (SECO) sur les transferts de fonds de la diaspora serbe, une autre étude menée par l'Ecole polytechique fédérale de Lausanne (EPFL) sur la diaspora dominicaine en Suisse et, finalement, un projet de collecte d'informations concernant la diaspora subsaharienne, dirigé par la Direction du développement et de la coopération (DDC).

11 Une première base de données, concernant les ressortissants colombiens, indiens et sud-africains, est en cours de création par l'EPFL (<http://cooperation.epfl.ch/ScientificDiasporasnetwork $>$ ); en outre, une banque de données des compétences africaines est en cours de création par l'association «Regards africains » à Genève (<http://regardsafricains.org>). 
ces chargés de projet et les populations bénéficiaires était tel qu'il pouvait nuire au bon déroulement des activités. Dans le même ordre d'idées, d'autres associations ont constaté que la collaboration avec des migrants issus des zones d'intervention des projets n'était pas toujours heureuse et créait parfois d'importantes difficultés. Cette constatation semblerait encore plus marquée lorsque le pays concerné est en conflit. Ainsi, une association développant des projets de promotion de la paix en collaboration avec les sociétés civiles israélienne et palestinienne explique qu'elle préfère ne pas établir de collaboration avec les associations de migrants de ces deux «pays » à Genève, et ce afin de préserver l'impartialité des projets. L'association en question, qui s'était posé d'abord la question de l'utilité d'une telle collaboration, avait conclu par expérience que le jeu n'en valait pas la chandelle. Cette réticence paraît liée surtout aux situations de conflit, mais l'existence de décalages socio-économiques entre la population bénéficiaire et les migrants établis à Genève peut aussi conduire à des divergences d'intérêts et s'avérer contre-productive pour les projets.

En revanche, d'autres associations membres de la FGC ont choisi de confier le suivi des actions à des ressortissants des régions bénéficiaires des projets, estimant que leur connaissance du terrain, leur expérience et leur motivation représentaient un réel avantage. De plus, deux tiers des ONG membres de la FGC comptent parmi leurs membres des ressortissants issus des régions dans lesquelles les projets sont développés.

\section{Un choix politique difficile}

La réflexion que la FGC a lancée en son sein sur les liens entre migration et développement implique que tôt au tard elle prenne position sur cette délicate question. En effet, le simple fait d'intégrer cette problématique dans ses préoccupations représente un acte politique. Dire, à contre-courant du contexte sociopolitique dominant, que la migration représente un réel potentiel, c'est une position politique. Il faut se donner les moyens de l'assumer.

En janvier 2008, dans le cadre du $2^{\text {e }}$ Carrefour de la solidarité, la FGC a voulu présenter la migration sous un jour positif et montrer à quel point le phénomène revenait à un échange de richesses entre deux mondes. Les migrants viennent en Suisse combler un manque de main-d'œuvre chronique, ils représentent un remède au vieillissement de nos sociétés et constituent un enrichissement sur le plan financier mais aussi culturel, pour le Nord et le Sud. Plus spécifiquement, les discussions ont porté entre autres sur les transferts de fonds et le soutien à certains projets d'associations de migrants qui peuvent avoir un effet positif sur le développement des pays d'origine.

Les partenaires du Sud qui se sont exprimés lors du Carrefour de la solidarité font toutefois une lecture beaucoup plus critique de la migration internationale, et les voix africaines ont été nombreuses à évoquer l'envers de la médaille de la migration, présentée comme la conséquence de l'échec de la politique gouvernementale de développement. Ainsi, Aminata Traoré, écrivaine et ancienne ministre de la Culture et du Tourisme du Mali, a fait remarquer que «personne ne se jetterait à la mer s'il y avait eu du développement. Jusqu'à présent, le développement a consisté à imposer des réformes néolibérales. Tout le problème vient du fait qu'on veut imposer ce modèle de société, avec l'aide de dirigeants qui ont choisi d'être 
de bons élèves du Fonds monétaire international (FMI) et de la Banque mondiale. Il n'y a eu aucun développement en Afrique. Il n'y a eu que du profit. Nos existences sont régies par les lois d'un marché qui ne nous ont jamais été favorables. $\gg^{12}$

C'est dans ce contexte que la société civile et les ONG doivent se positionner et faire des choix en lien avec cette question de la migration et du développement. Pour les ONG de développement du Nord, toute prise de position politique dans ce débat est très délicate. Il faut être attentif, d'un côté, à ne pas légitimer le discours d'une certaine droite populiste qui soutiendrait la coopération au développement si celle-ci permettait réellement de mettre un frein à la migration et, de l'autre côté, il faut réussir à évoquer la migration sous son jour positif, sans toutefois passer outre le fait que la «migration du désespoir» pousse chaque année des milliers de corps noyés sur les plages de l'Europe et fragilise les relations parents-enfants de ceux et de celles qui laissent leurs enfants au pays pour venir travailler en Europe - justement pour le bien de leur famille ${ }^{13}$. Il est intéressant aussi de rappeler les études récentes qui ont mis en lumière le phénomène du migration hump, qui montre que le développement peut, dans un premier temps, favoriser la migration.

Lors de son intervention au $2^{\mathrm{e}}$ Carrefour de la solidarité, Aminata Traoré a également mis en doute le terme «migration» lui-même: pourquoi les 600000 Suisses de l'étranger ne sont-ils pas appelés «migrants », à l'instar de tous les ressortissants étrangers au Nord? Cela démontre clairement, selon Aminata Traoré, l'utilisation discriminatoire du terme «migrant» par les pays du Nord.

Pour certains, de nombreux acteurs en Suisse discutent aujourd'hui de la thématique de la migration par opportunisme. Dans un contexte politique très critique envers l'aide au développement, celle-ci tend à se restreindre à une vision utilitaire: un outil pour améliorer les conditions de vie des populations défavorisées qui ainsi ne seront pas tentées par l'émigration en Europe. Les œuvres d'entraide doivent veiller à ce que la coopération suisse ne soit pas instrumentalisée et ne devienne pas qu'un outil pour promouvoir les intérêts de la Suisse. La situation s'est complexifiée davantage depuis le durcissement des lois sur l'asile et les étrangers, et cette question politique interne doit être abordée par les associations de migrants et de défense des migrants. La FGC et les organisations de solidarité internationale doivent aussi empoigner le débat à leur niveau en présentant les opportunités liées à la migration, sans l'idéaliser, et en favorisant les possibilités de maximiser ces effets bénéfiques sur les sociétés du Sud. Dans cette problématique extrêmement complexe et sensible, la société civile se doit d'occuper le terrain et de proposer des solutions concrètes tout en continuant d'alimenter la réflexion et les échanges en la matière.

En définitive, c'est peut-être en termes de choix pour les populations que les résultats en matière de développement seront mesurés: choix de décider librement de migrer ou non, sans être poussé par la pauvreté ou le désespoir vers des terres lointaines, à la recherche d'un très hypothétique avenir meilleur.

12 Voir aussi le plus récent ouvrage d'Aminata Traoré, L'Afrique humiliée, Paris, Fayard, 2008.

13 Une représentante du Collectif de soutien aux sans-papiers de Genève s'est demandé, lors d'un atelier organisé lors du $2^{\mathrm{e}}$ Carrefour de la solidarité, si son effort en valait la peine: «Avec ses enfants, on ne rattrape jamais ces années perdues. » 